\title{
MAGNETIC FIELD DEPENDENT EFFECTIVE MASS IN THE ORGANIC SUPERCONDUCTOR
}

$$
\kappa-(\mathrm{BEDT}-\mathrm{TTF})_{2} \mathrm{I}_{3}
$$

\author{
E. Balthes, D. Schweitzer.
}

3. Physik. Institut, Universität Stuttgart, Pfaffenwaldring 57, 70550 Stuttgart, Germany

I. Heinen, H.J. Keller

Anorg.-Chemisches Institut, Universität Heidelberg

Im Neuenheimer Feld 270, 69120 Heidelberg, Germany

W. BibERACHER

Walther Meissner Institut,. Walther-Meissner-Str. 8, 85748 Garching, Germany
A.G.M. Jansen and E. STEeP

High Magnetic Field Laboratory, MPI für Festkörperforschung 38042 Grenoble Cedex 9, France

$\kappa$-(BEDT-TTF $)_{2} \mathrm{I}_{3}$ is an electronically extreme two-dimensional organic metal with a superconducting transition at around $4 \mathrm{~K}$. In magnetic fields above $12 \mathrm{~T}$ the effective mass, as obtained from the temperature dependence of the amplitudes of Shubnikov-de Haas oscillations, is magnetic field dependent as long as the magnetic field is arranged perpendicular to the conducting planes. In contrast to this, by turning the magnetic field by $27^{\circ}$ or even only $9^{\circ}$ the observed effective mass (as obtained from Shubnikovde Haas and de Haas-van Alphen measurements) is field independent. We suppose that the occurrence of anyons at temperatures below $1 \mathrm{~K}$ in fields above $12 \mathrm{~T}$ is the reason for the observed field dependence of the effective mass.

PACS numbers: $74.70 . \mathrm{Kn}, 71.25 . \mathrm{Hc}, 71.25 . \mathrm{Jd}$

\section{Introduction}

The highest superconducting transition temperatures $\left(T_{\mathrm{c}}\right)$ in the class of the organic superconductors are observed in the so-called $\kappa$-phases of radical salts of the donor BEDT-TTF [bis-(ethylenedithiolo)-tetrathiafulvalene]. Here, among the ambient pressure superconductors, $\kappa$-(BEDT-TTF $)_{2} \mathrm{Cu}(\mathrm{NCS})_{2}$ possesses the second highest $T_{\mathrm{c}}$ of $10.4 \mathrm{~K}[1]$ and $\kappa$-(BEDT-TTF $)_{2} \mathrm{CuN}(\mathrm{CN})_{2} \mathrm{Br}$ the highest $T_{\mathrm{c}}$ of $12.2 \mathrm{~K}$ [2]. In those $\kappa$-phases the donor molecules are arranged in sheets and form face-to-face orthogonal dimers. There are two dimers per unit cell, related to each other by a $2_{1}$ screw rotation. Taken by itself, without the anions, the donor layer is essentially centrosymmetric. The donor molecule packing in $\kappa$-(BEDT-TTF $)_{2} \mathrm{I}_{3}\left(T_{\mathrm{c}} \approx 4.0 \mathrm{~K}\right)[3,4]$ is very similar with respect to 
$\kappa$-(BEDT-TTF $)_{2} \mathrm{Cu}(\mathrm{NCS})_{2}$, however, the space group is centrosymmetric $\left(P 2_{1} / c\right)$, allowing the $\mathrm{I}_{3}$ anions to be located on inversion centers. Due to the centrosymmetric space group of $\kappa$-(BEDT-TTF $)_{2} I_{3}$ there exists a difference in the Fermi surface with respect to $\kappa$-(BEDT-TTF $)_{2} \mathrm{Cu}(\mathrm{NCS})_{2}$. Using a tight binding extended Hückel method the band structures and Fermi surfaces of $\kappa$-(BEDT-TTF $)_{2} \mathrm{Cu}(\mathrm{NCS})_{2}$ and $\kappa$-(BEDT-TTF $)_{2} \mathbf{I}_{3}[5,3]$ were calculated and in the case of $\kappa$-(BEDT-TTF $)_{2} \mathrm{I}_{3}$ two conduction bands are found which are degenerated along the $Z-M$ zone boundary, leading to nearly circular hole orbits around $Z$ and around $\Gamma$ (see Fig. 1 insert). In $\kappa$-(BEDT- TTF $)_{2} \mathrm{Cu}(\mathrm{NCS})_{2}$ two bands cross the Fermi level as well, but the degeneracy is removed and the corresponding orbit around $\Gamma$ now is open. In fact, those details of the Fermi surfaces were observed by Shubnikov-de Haas (SdH) and de Haas-van Alphen (dHvA) measurements $[6,7]$. While in $\kappa$-(BEDT-TTF $)_{2} \mathrm{Cu}(\mathrm{NCS})_{2}$ in low magnetic fields only one oscillation frequency in the SdH and dHvA measurements is observed and the orbit around $\Gamma$ occurs only as a magnetic breakdown effect in fields above $15 \mathrm{~T}$ [7], in the $\mathrm{SdH}$ and dHvA measurements of $\kappa$-(BEDT-TTF) ${ }_{2} \mathrm{I}_{3}[6]$ two oscillation frequencies (at $F_{1}=575 \mathrm{~T}$ and $F_{2}=3879 \mathrm{~T}$ ) are observable. In cases where the applied magnetic field is arranged perpendicular to the conducting planes in $\kappa$-(BEDT-TTF $)_{2} \mathrm{I}_{3}$, from the temperature dependence of the $\mathrm{SdH}$ and $\mathrm{dHvA}$ oscillation amplitudes constant values for the effective mass in the magnetic field range between $6 \mathrm{~T}$ and $12 \mathrm{~T}$ were found $\left(m^{*}=3.9 m_{0}\right)$.

The large oscillation amplitude of $F_{2}$ at fields above $9 \mathrm{~T}$ over a considerable temperature range allows the determination of the effective mass with high accuracy so that it is possible to find out whether $m^{*}$ is magnetic field dependent or not. A magnetic field dependent effective mass might be observed in cases where the conventional independent particle mass is enhanced due to many-body interactions such as electron-phonon or electron-electron interactions. In the electronically extreme 2D system $\kappa$-(BEDT-TTF) ${ }_{2} \mathrm{I}_{3}$ (see Refs. $[4,7$ and 9$]$ ) other typical 2D phenomena such as the occurrence of anyons at low temperatures and in very high magnetic fields might lead to a magnetic field dependent effective mass as well.

\section{Experimental}

$\kappa$-(BEDT-TTF $)_{2} \mathrm{I}_{3}$ was first prepared by Kajita et al. [3]. In their preparation mixed electrolytes of $\left(n-\mathrm{C}_{4} \mathrm{H}_{9}\right)_{4} \mathrm{NI}_{3}$ and $\left(n-\mathrm{C}_{4} \mathrm{H}_{9}\right)_{4} \mathrm{NAuI}_{2}$ were used, resulting in crystals of $\kappa$-(BEDT-TTF $)_{2}\left(\mathrm{I}_{3}\right)_{1-x}\left(\mathrm{AuI}_{2}\right)_{x},(x \approx 0.006)$. In our preparation, which was similar as reported for $\alpha$-phase crystals [8], we used only the $\left(n-\mathrm{C}_{4} \mathrm{H}_{9}\right)_{4} \mathrm{NI}_{3}$ salt in order to obtain pure, high quality single crystals of $\kappa$-(BEDT-TTF $)_{2} \mathrm{I}_{3}$. The unit cell parameters for those monoclinic single crystals $\left(P 2_{1} / c\right)$ are: $a=16.429 \AA$, $b=8.504 \AA, c=12.876 \AA, b=108.50^{\circ}, V=1705.97 \AA^{3}$ and $R_{\mathrm{W}}=0.042$ (here we have used the same convention as in Refs. [3] and [7] in contrast to Ref. [4] where the space group $P 2_{1} / a$ was employed). These parameters are slightly different from those obtained by Kajita et al. [3]. Crystals used in our experiments typically had dimensions of $2 \times 1 \times 0.5 \mathrm{~mm}^{3}$ and superconducting transition temperatures of $\approx 4.0 \mathrm{~K}$ (measured by resistivity). The resistivity measurements were performed by the usual four-point method. $\mathrm{SdH}$ and $\mathrm{dHvA}$ measurements were done at the 
High Field Laboratory of the Max Planck Institut in Grenoble, in magnetic fields up to $27 \mathrm{~T}$ and in the temperature range $0.4-1.3 \mathrm{~K}$. The crystals were mounted in a holder so that they could be tilted (during the experiment) around their $b$ - and $c$-axis, the initial field orientation being along the $a^{*}$-axis (normal to the $b c$-plane of conduction). The resistivity was always measured along the $c$-direction. The dHvA experiments were performed in the usual torque arrangement. In order to obtain sufficient large dHvA signals, the magnetic field had to be turned out of the direction perpendicular to the conducting planes by $27^{\circ}$. In these cases the obtained $m^{*}\left(\Theta=27^{\circ}\right)$ had to be multiplied by $\cos 27^{\circ}=0.891$ in order to obtain the value $m^{*}\left(\Theta=0^{\circ}\right)$. At $25 \mathrm{~T}$ the magnetic field was turned only by $9^{\circ}$.

\section{Results}

On several single crystals of $\kappa$-(BEDT-TTF) ${ }_{2} I_{3} \mathrm{SdH}$ and dHvA experiments were performed in magnetic fields up to $27 \mathrm{~T}$. Figure 1 shows typical dHvA and $\mathrm{SdH}$ oscillations obtained from those experiments. Giant quantum oscillations can

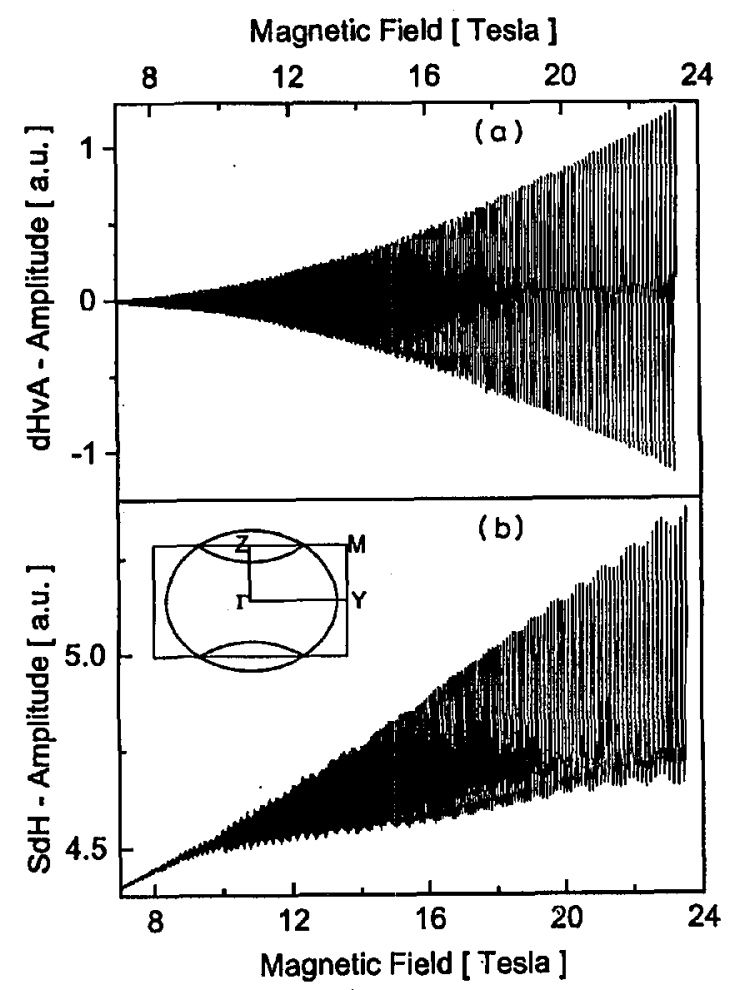

Fig. 1. (a) dHvA oscillations and (b) $\mathrm{SdH}$ oscillations of a single crystal of $\kappa$-(BEDT-TTF $)_{2} \mathrm{I}_{3}$ at $0.4 \mathrm{~K}$ versus the magnetic field. Insert: Fermi surface of $\kappa-(\mathrm{BEDT}-\mathrm{TTF})_{2} \mathrm{I}_{3}$. 


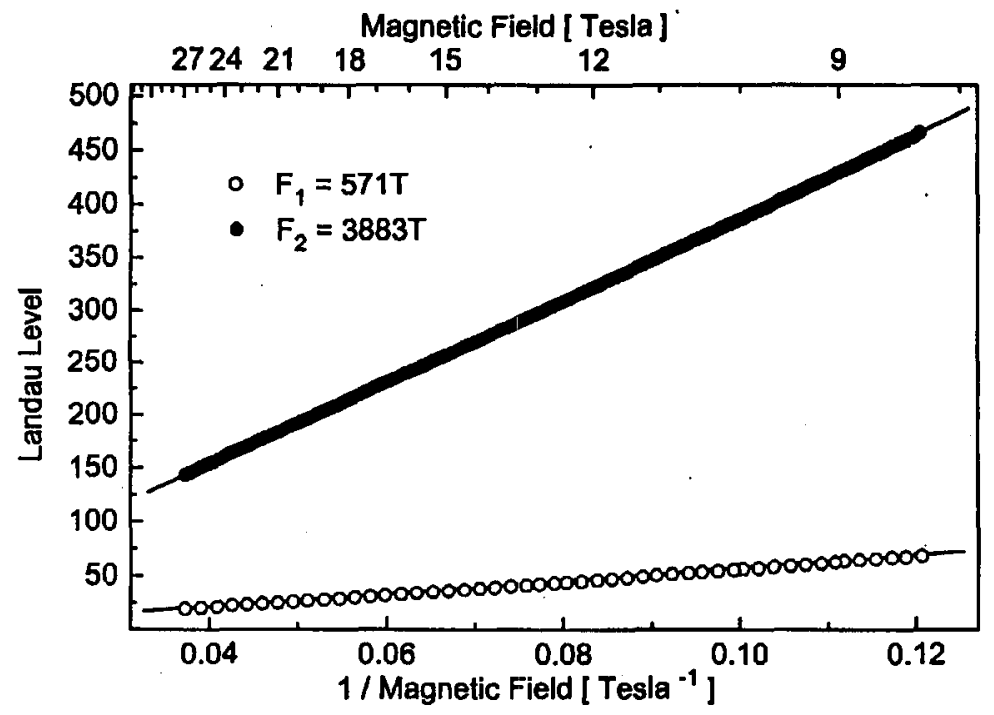

Fig. 2. Landau levels of both oscillation frequencies $F_{1}$ and $F_{2}$ of $\kappa$-(BEDT-TTF $)_{2} \mathrm{I}_{3}$ versus the inverse magnetic field.

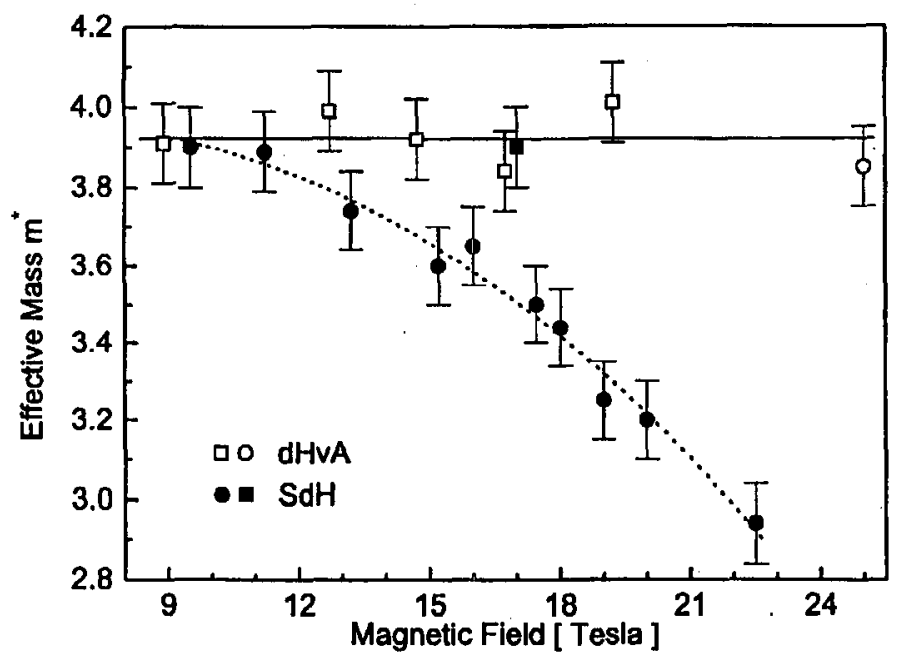

Fig. 3. Magnetic field dependence of the effective mass $m^{*}$ for the larger orbit $\left(F_{2}\right)$ of the Fermi surface of $\kappa$-(BEDT-TTF $)_{2} \mathrm{I}_{3}$, as obtained from the temperature dependence of the $\mathrm{SdH}$ and $\mathrm{dHvA}$ oscillation amplitudes (see text).

be seen. At high magnetic fields the SdH oscillation amplitude is of the order of $20 \%$ with respect to the nonoscillating part of the magnetoresistivity. The insert in Fig. 2 shows the Fermi surface of $\kappa$-(BEDT-TTF) $)_{2} \mathrm{I}_{3}$ [3]. 
In Fig. 2 the number of the Landau levels (for $F_{1}$ and $F_{2}$ ) versus the reciprocal field $1 / B$ for $B \| \boldsymbol{a}^{*}$ is drawn. From the slope of the plots more precise values of $F_{1}=571 \mathrm{~T}$ and $F_{2}=3883 \mathrm{~T}$ are obtained. With the assumption of a circular Fermi surface, i.e. $\varepsilon_{\mathrm{F}}=h^{2} A_{k} / 8 \pi^{3} m_{0}$, where $\varepsilon_{\mathrm{F}}$ is the Fermi energy and $A_{k=2}=8 \pi^{3} e F_{2} / h$ is the area of the Fermi surface corresponding to $F_{2}$, the Sommerfeld coefficient for a 2D electron gas $\gamma=\pi^{2} R k_{\mathrm{B}} / 3 \varepsilon_{\mathrm{F}}=(20.5 \pm 0.5) \mathrm{mJ} \mathrm{K}^{-2} \mathrm{~mol}^{-1}$ is obtained in excellent agreement with a value of $(18.9 \pm 2) \mathrm{mJ} \mathrm{K}^{-2} \mathrm{~mol}^{-1}$ obtained by a specific heat measurement [9].

From the SdH and the dHvA experiments the effective masses at several magnetic fields were determined from the temperature dependence of the oscillation amplitudes using the Lifshitz-Kosevich formula. In addition one value of $m^{*}$ was determined from an $\mathrm{SdH}$ experiment at about $17 \mathrm{~T}$ under an angle $\Theta=9^{\circ}$. Figure 3 shows the effective masses obtained from both types of experiments.

\section{Discussion}

The angular dependence of the higher oscillation frequency as well as of the effective mass at $12 \mathrm{~T}$ (see Refs. [6a and 9]) perfectly follows the $(1 / \cos \theta)$-law as expected for a cylindrical Fermi surface of a 2D electronic system. Furthermore, from $\mathrm{dHvA}$ experiments at $12 \mathrm{~T}$ and measurements of the specific heat [9] it was shown that up to $12 \mathrm{~T} \kappa$-(BEDT-TTF $)_{2} \mathrm{I}_{3}$ can be described very well by the $2 \mathrm{D}$ free electron model. Therefore, it is surprising that for $\Theta=0^{\circ}$ in fields above $12 \mathrm{~T}$ the effective mass $m^{*}$ continuously decreases with increasing magnetic field. In contrast from the dHvA experiments at $\Theta=27^{\circ}$ and $\Theta=9^{\circ}$ in the whole field range as well as from the $\mathrm{SdH}$ experiment at $\Theta=9^{\circ}$ at $17 \mathrm{~T}$ a magnetic field independent $m^{*}\left(0^{\circ}\right)=3.9 m_{0}$ (the same value as from all measurements below $12 \mathrm{~T}$ ) is obtained. Since dHvA experiments are made at $\Theta=27^{\circ}$ and $\Theta=9^{\circ}$, the electrons have to leave the individual conducting plane they belong to and cross several planes (at $25 \mathrm{~T}$ at least 10 planes) following their cyclotron orbits perpendicular to the direction of the magnetic field. In this case we detect the behavior of a $2 \mathrm{D}$ electronic system in three dimensions. In contrast in $\mathrm{SdH}$ experiments the magnetic field is arranged exactly perpendicular to the conducting planes so that the cyclotron orbits lie completely within the individual conducting planes. In this case we investigate a $2 \mathrm{D}$ electronic system under broken time-reversal symmetry and broken parity so that all the conditions for the occurrence of anyons [10], quasi-particles which can only exist in a $2 \mathrm{D}$ electronic system, are fulfilled. Since anyons do not obey Fermi statistics, we suppose that the occurrence of anyons leads to a decrease in the SdH oscillation amplitudes at high magnetic fields and temperatures below $1 \mathrm{~K}$ and therefore from the fit of the experimental data to the LK-formula smaller effective masses are obtained. In fact, those obtained effective masses are not the real effective masses, because the LK-formula, which is valid only for electronic systems with a constant number of fermions, cannot be used here for the determination of the effective mass in contrast to the dHvA and SdH experiments at angles $\Theta>0^{\circ}$. Therefore, we do not directly observe the anyons in our experiment, but a decreasing SdH oscillation amplitudes due to the occurrence of anyons at $\Theta=0^{\circ}$. 


\section{Acknowledgments}

Very stimulating and valuable discussions with Professors Yu.A. Bychkov, A.M. Kosevich, T. Maniv, I. Wagner, M. Weger and Dr. J. Wosnitza are gratefully acknowledged. This work was supported by the Deutsche Forschungsgemeinschaft, the Forschungsschwerpunkt Supraleitung des Landes Baden-Württemberg and by the Commission of the European Communities [contract CI-CT 90-0863 (CD)].

\section{References}

[1] H. Urayama, H. Yamochi, G. Saito, K. Nozawa, T. Sugano, M. Kinoshita, S. Sato, K. Oshima, A. Kawamoto, J. Tanaka, Chem. Lett. 1988, 55 (1988).

[2] J.M. Williams, A.J. Schultz, U. Geiser, K.D. Carlson, A.M. Kini, H.H. Wang, W.-K. Kwok, M.-H. Whangbo, J.E. Schirber, Science 252, 1501 (1991).

[3] K: Kajita, Y. Nishio, S. Moriyama, W. Sasaki, R. Kato, H. Kobayashi, A. Kobayashi, Solid State Commun. 64, 1279 (1987).

[4] E. Balthes, A. Breining; S. Kahlich, J. Moldenhauer, D. Schweitzer, P. Bele, H. Brunner, I. Heinen, B. Nuber, H.J. Keller, Synth. Met. 56, 2859 (1993).

[5] K. Oshima, T. Mori, H. Inokuchi, H. Urayama, H. Yamochi, G. Saito, Phys. Rev. $B$ 38, 938 (1988).

[6] (a) M. Heinecke, K. Winzer, D. Schweitzer, Z. Phys. B 93, 45 (1993); (b) E. Balthes, D. Schweitzer, I. Heinen, H.J. Keller, W. Biberacher, A.G.M. Jansen, E. Steep, Verh. der DPG, Münster 1994, p. 1665; (c) J. Wosnitza, N. Herrmann, X. Liu, D. Schweitzer, Synth. Met., in press.

[7] C.-P. Heidmann, H. Müller, W. Biberacher, K. Neumaier, C. Probst, K. Andres, A.G.M. Jansen, W. Joss, Synth. Met. 42, 2029 (1991).

[8] K. Bender, I. Hennig, D. Schweitzer, K. Dietz, H. Endres, H.J. Keller, Mol. Cryst. Liq. Cryst. 108, 359 (1984).

[9] J. Wosnitza, X. Liu, D. Schweitzer, H.J. Keller, Phys. Rev. B, in press.

[10] F. Wilczek, Phys. Rev. Lett. 49, 957 (1982). 